\title{
Pain-Mapping in Musculoskeletal Ultrasound - A Pilot Study with Initial Observations
}

\section{Cormick $\mathbf{W}^{1 *}$ and McGregor $\mathbf{R}^{2}$}

${ }^{1}$ Canberra Specialist Ultrasound Canberra, Australian Capital Territory, Australia

${ }^{2}$ Director USVASC, Australian Capital Territory, Australia

*Corresponding author: Wes Cormick BMed, BMedSc, FRACP, DDU, Australian Capital

\section{Opinion}

Volume 4 Issue 3

Received Date: August 15, 2020

Published Date: September 07, 2020

DOI: $10.23880 /$ crij-16000177

\section{Abstract}

In addition pain-mapping allows quantification of the study giving the opportunity for assessment and documentation of change in progress studies.

Our early experience with pain-mapping has already revealed several novel concepts in MSK imaging which have led to altered treatments for these conditions:

- Our experience with pain-mapping of tendinopathy and tendon tears shows the pain rarely comes from the tear itself but rather the adjacent abnormal tendon.

- Pain-mapping of achilles tendon, and patellar tendon pathology suggests that the paratenon is an important source of pain.

- In the painful shoulder, pain-mapping reveals the role of coracoid enthesopathy.

- In lateral hip pain, pain-mapping has revealed the importance of the piriformis tendon in contributing to symptoms.

- In the case of chronic athletic groin pain, pain-mapping reveals different patterns between individuals who have presented with essentially common clinical signs and provides the option of more individualised treatment.

- Widespread high pain-mapping scores with no abnormality on imaging can suggest a diagnosis of fibromyalgia.

\section{Introduction}

In musculoskeletal ultrasound, the usual clinical question is to find the cause of a patient's pain.

To elaborate on this we would like to introduce the concept of:

- Actual pathology

- Sonopathology

- Pathodynia (painful pathology)

The relationship between these is shown by Venn diagram (Figure 1).

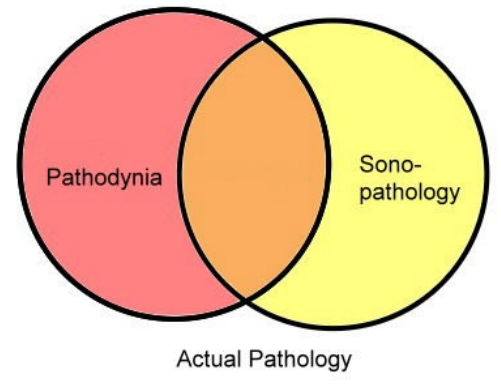

Figure 1: A Venn diagram showing the relations between actual pathology, sonopathology and pathodynia. 


\section{Clinical Radiology \& Imaging Journal}

Pathology that is painful but does not show up on ultrasound (red).

Pathology which is painful and does show up on ultrasound (orange).

Pathology which shows up on ultrasound but is not clinically relevant (yellow).

Actual Pathology: Pathology is present but not always demonstrable with ultrasound. This can include mild tendinosis, chronic ligament strains, fat pad inflammation and nerve compression/inflammation.

Sonopathology: Pathology seen with ultrasound which might or might not be symptomatic. Asymptomatic sonopathology includes quite a range of actual pathology and includes tendon tears, tendinosis, thickened bursae, calcium deposits and old muscle tears.

Pathodynia: (painful pathology) is what we are asked to find with the ultrasound study.

We are asked to differentiate between:

- Pathology that is painful but does not show up on ultrasound (red).

- Pathology which is painful and does show up on ultrasound (orange).

- Pathology which shows up on ultrasound but is not clinically relevant (yellow).

\section{Pain-Mapping Involves Needling the Tissues} to See if They are Tender Or Not Which Allows Us to Differentiate These Three Groups

\section{How is it Done}

Pain-mapping is a simple procedure and with practice only adds a few minutes to the study.

Under ultrasound guidance a fine needle gently probes around the symptomatic soft tissues as indicated by the patient.

Wide sampling is performed based on the clinical presentation and regardless of the ultrasound findings. The patient scores each site on an analogue pain scale of 1 to 10 and also comments if the pain is similar or different to that which has caused them to present. This allows the construction of a 'pain map' highlighting those soft tissues contributing to the patient's pain.

We find that in athletes, the elderly and in postoperative patients there are often multiple pathologies on imaging and pain mapping allows us to determine which are most symptomatic.

\section{Methods}

We collected 100 consecutive pain-mapping cases over a two and a half month period, working 3 days a week. In a busy tertiary referral MSK ultrasound practice we performed on average three and a half cases a day, showing that the majority of cases did not require pain-mapping. In all cases there were 2 operators in the room one doing the needling and an assistant recording the pain scores by site.

\section{A Number of Novel and Interesting Ideas Came from Our Observation of These 100 Cases}

\section{The Errancy of Imaging}

The first striking finding is the fallibility of imaging.

In our experience many radiologists and sonographers come to believe in the infallibility of imaging. The results of this study and questioning of our referring doctors, reveals however that MSK imaging is imperfect and frequently frankly wrong as to the cause of pain. When compared to the pain map result, imaging was correct only $80 \%$ of the time. Conversely our clinical impression from history and physical exam was correct $98 \%$ of the time.

This suggests we should emphasise clinical assessment as part of the MSK ultrasound exam and this should be reflected in the teaching our colleges of the primacy of clinical features and fallibility of imaging.

Few radiologists have a problem with a statement at the end of a mammogram or breast ultrasound study which states the study is fallible and may miss breast cancers. We suggest the same condition applies to MSK ultrasound studies. Although MRI was not assessed in this study 23 patients also had MRI of the region and it seems to have a similar accuracy when compared to pain-mapping.

\section{Where does Tendon Pain Come From?}

Pain-mapping of torn tendons shows that old tendon tears rarely hurt. The act of a tendon tearing may hurt but subsequent pain comes from the tendinopathy surrounding the tear, not the tear itself.

In tendinopathy the end stage tenomalacia usually does not hurt. In many patients the pain is associated with tendon with relatively normal appearance. This suggests there are 


\section{Clinical Radiology \& Imaging Journal}

microscopic pathological changes that cause tendon pain but do not cause any findings on ultrasound. From the small cohort who have also had MRI, this also holds true for that modality.

In typical established patellar tendinopathy there is a hypoechoic region in the deep proximal portion of the tendon. This portion is not tender but the more normal looking tendon around it is.

In both Achilles tendon pain and patellar tendon pain, there is a significant component of pain coming from the paratenon, typically about $50 \%$ of the overall pain. This has lead us to alter our treatment for these tendons with a hydrostatic paratenon stripping with cold normal saline and steroid. We use cold solution to try and inhibit bleeding from the small disrupted vessels. We may also add polidocanol into the tendon which can be done at the same attendance, unlike PRP (platelet rich plasma) which cannot be done if using steroid around the tendon. We have done cold saline stripping on its own with PRP to prevent the steroid inhibiting the action of the PRP.

IF both paratenon steroid and PRP are to be used we wait 7 - 10 days between procedures.

We believe the aim of injection therapy is to relieve pain in the tendon to allow them to better comply with their physical therapy programs.

\section{Coracoid Enthesopathy}

In assessing patients with degenerative cuff disease (spectrum of subdeltoid bursitis, rotator cuff tendinopathy/ enthesopathy/tears) we found the $40 \%$ had a painful coracoid process.

In patients presenting with anterior shoulder pain there was $96 \%$ incidence of coracoid pain. We pain map directly over the coracoid process and along the 5 main attachments to the coracoid process. In patients who we were seeing for incomplete response to therapy elsewhere most had a painful coracoid as the cause of their incomplete response to therapy.

Nuclear medicine bone scan may show increased uptake of the coracoid process in patients with degenerative cuff disease or degenerative joint disease of the shoulder (Figure 2). We treat this with injection of steroid and local anaesthetic over the coracoid and along any of the attaching ligaments or tendon if they are significantly painful on mapping. We then arrange post injection physical therapy to the attaching structures if they were painful.

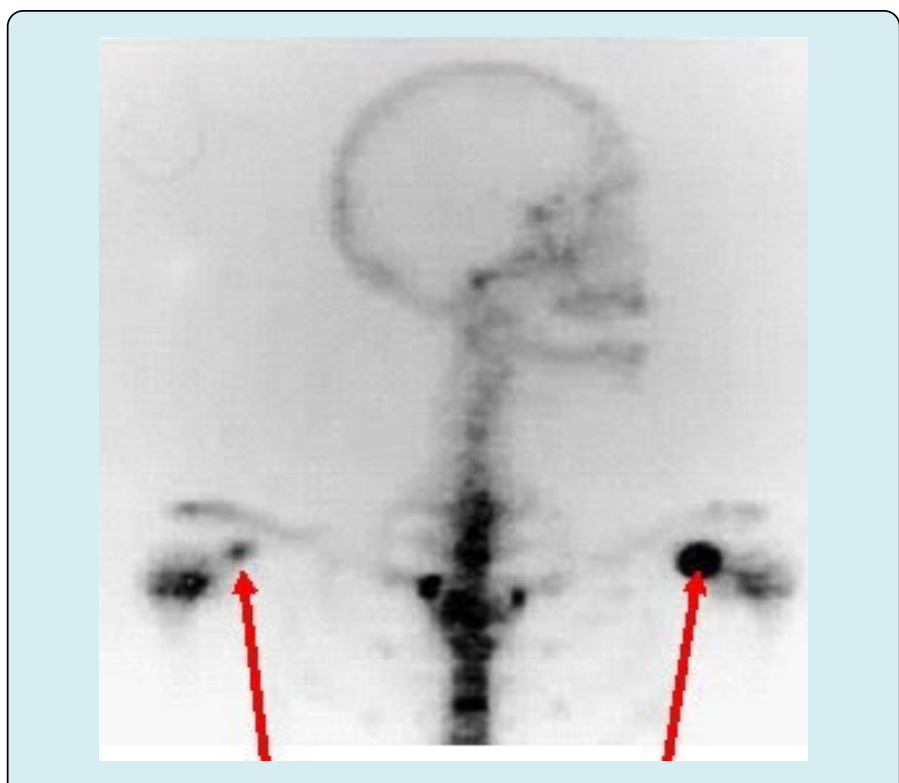

Figure 2: A nuclear medicine bone scan showing intense uptake at the coracoid process bilaterally consistent with active enthesopathy.

The ultrasound appearance of active coracoid enthesopathy includes a hypoechoic soft tissue line adjacent to the bony process and sometimes erosions or irregularity in the underlying bone contour (Figure 3).

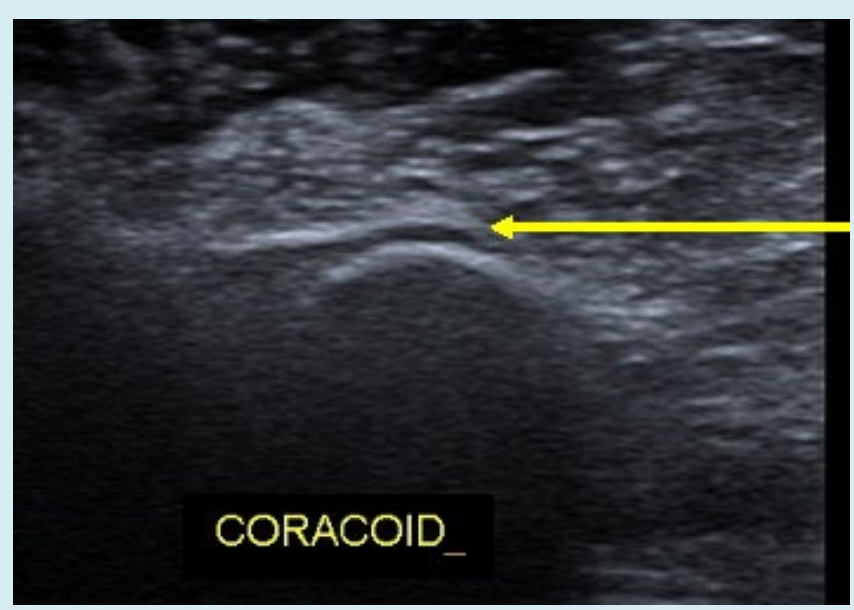

Figure 3: An ultrasound directly over a painful coracoid process showing the hypoechoic change of enthesopathy directly adjacent to the bony process.

\section{Piriformis Enthesopthy}

Greater Trochanter Pain Syndrome (GTPS) is thought to be due to the enthesis organ of the three gluteal tendons. That is the tendons, enthesis and corresponding bursae of the 
gluteus maximus, gluteus medius and gluteus minimus. We found that $92 \%$ of GTPS patients had significant pain scores from the piriformis tendon and its enthesis as well (Figure 4).

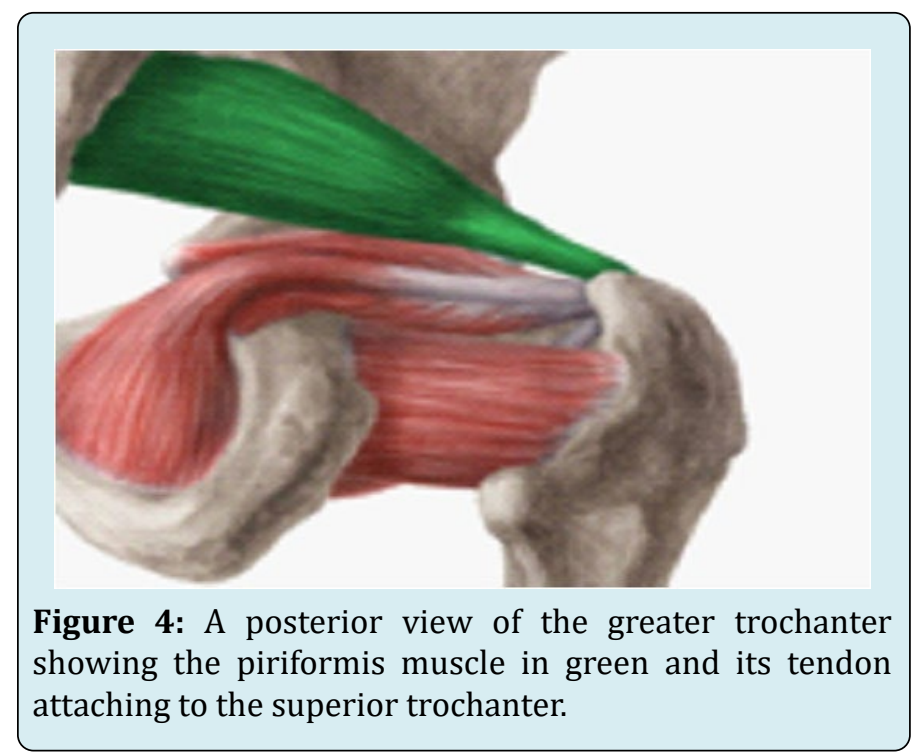

In patients who had an injection of the usual gluteal entheses and a good response, but recurrence of lateral hip pain, we found most had a painful tear in the medius or minimus muscle from rushing their physical therapy.

Patients who were injected but had an incomplete response usually had significant ongoing pain from the untreated piriformis tendon.

\section{Progress Studies}

The GTPS cases above show how pain-mapping can be useful for progress assessment. As well as just quantifying the pain level it can be used to sort out the cause of recurrence of symptoms post-injection. There are 3 common patterns.

The patient comes in with painful condition (A) and it responds well to injection (a) but recurs and is painful again $\left(A^{\prime}\right)$.

$$
A \rightarrow a \rightarrow A^{\prime}
$$

The patient presents with condition (A), it responds well to injection (a) and a similar but slightly different pain comes on (B). An example of this is the tear in the gluteal muscle belly as described above.

$$
\mathrm{A} \rightarrow \mathrm{a} \rightarrow \mathrm{B}
$$

The patient presents with complex condition (AB) and only one aspect of it is treated leaving one aspect still painful $(\mathrm{aB})$. An example of this which we see is where the piriformis is not treated in GTPS or the coracoid is not treated in shoulder pain.

$$
\mathrm{AB} \rightarrow \mathrm{aB}
$$

\section{Groin Pain}

Chronic groin pain in athletes is controversial and complex. Particularly those with refractory post- surgical pain. In the small number of groin pain cases we have pain-mapped we found each to be quite different. The soft tissue structures of the groin are a complex enthesis and pain mapping has allowed us to show which structures were painful in each case despite weaknesses of the walls, tears, and previous surgery. Only 3 have been done so far but it appears to be a promising technique for unravelling this complex area and help target conservative and surgical treatments (Figure 5).

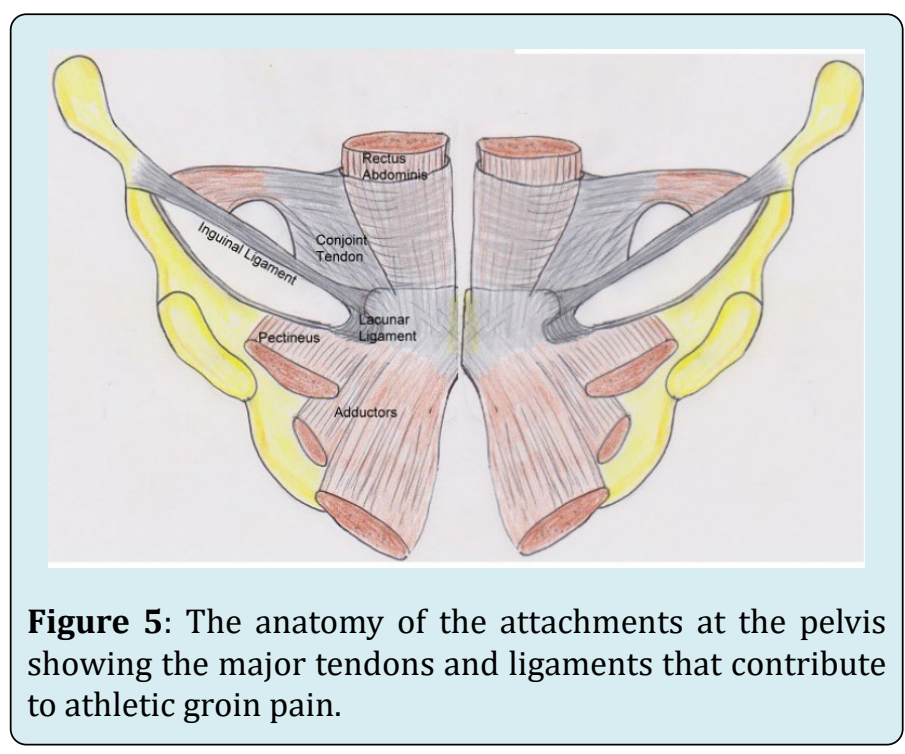

\section{Fibromyalgia}

There was a single patient who had bilateral GTPS and had scores of $8-10$ for all muscle groups, tendons, bursae and entheses with perfectly normal imaging. This led us to suspect fibromyalgia which was confirmed on further investigation.

\section{Postoperative Pain}

There were several cases of post-operative pain where the surgeon wanted to know if the patient's pain was coming from the surgical site or somewhere else. In all cases the pain-mapping could distinguish the causes of pain as coming from the surgical site or if it was nearby pathology. 


\section{Clinical Radiology \& Imaging Journal}

\section{Who don't we Pain Map}

There were a number of cases where pain-mapping may very well have been useful but we decided not to progress the study to pain-mapping for a variety of reasons.

1. Straight forward cases

2. No sonographer/assistant in room

3. Children

4. Reduced I.Q.

5. Poor English language skills

6. Already struggling with pain levels

7. ? Secondary Gain -(e.g. workers compensation cases)

\section{Conclusion}

Pain-mapping in Musculoskeletal Ultrasound can increase the ability of an ultrasound study to detect painful pathology, particularly when the imaging component is unhelpful.
Several novel ideas have come out of this study which requires further assessment and scrutiny. These ideas may have a profound impact on future treatments of these conditions.

- The fallibility of imaging compared to clinical assessment.

- Tendon tears are rarely the painful

- The paratenon is an important source of pain in Achilles and patellar tendinopathy.

- Coracoid enthesopathy is an important cause of pain in degenerative joint disease and degenerative cuff disease of the shoulder.

- Piriformis enthesopathy is an important cause of pain in greater trochanter pain syndrome.

- In chronic athletic groin pain, there might be a wide variety of pathologies causing common clinical presentations. Treatments such as surgery could be tailored to the findings. 